\title{
Comparison of Wireless Network Penetration Testing Tools on Desktops and Raspberry Pi Platforms
}

\author{
Aparicio Carranza, $\mathrm{PhD}^{1}$, Daniel Mayorga, BTech ${ }^{1}$, Casimer DeCusatis, $\mathrm{PhD}^{2}$ and Hossein Rahemi, $\mathrm{PhD}^{3}$ \\ ${ }^{1}$ New York City College of Technology - CUNY, Brooklyn, NY USA, acarranza@citytech.cuny.edu \\ ${ }^{2}$ Marist College, Poughkeepsie, NY USA, casimer.decusatis@marist.edu \\ ${ }^{3}$ Vaughn College of Aeronautics \& Technology, East Elmhurst, NY USA, hossein.rahemi@vaughn.edu
}

\begin{abstract}
Wireless networks have become ubiquitous due to their ease of use and facilitation of mobile devices such as smart phones, tablets, and various Internet of Things (IoT) applications. This has driven a need for more advanced wireless penetration testing techniques, and for more technical professionals trained in wireless security. In this paper, we investigate three popular open source wireless penetration testing tools (Aircrack -ng, Reaver, and Kismet) and compare their behavior on a traditional desktop computer and a Raspberry Pi model 3. Use cases include packet sniffing and decryption of WEP, WPA, and WPA2 passwords. Based on this work, we make recommendations for using specific tools in cybersecurity training and education.
\end{abstract}

Keywords-WLAN, Kali Linux, Raspberry Pi, Kismet, Reaver

\section{INTRODUCTION}

In recent years, wireless local area networks (WLANs) have become widely adopted for a wide range of applications, from personal home networks to Fortune 500 enterprise class applications [1-4]. There has been a corresponding increase in the number and severity of wireless security issues, and a need for increased penetration testing on WLANs. At the same time, there has arisen a need for more education and practitioner training in wireless cybersecurity. Both professional penetration testers and education institutions have found that open source tools, such as those provided in Kali Linux [1-2], have an important role to play in securing modern networks. In fact, since many bad actors use these tools for network reconnaissance, sniffing, and decryption attacks, it makes sense to develop an ethical hacking framework around this toolkit. The widespread availability of low cost, mobile compute platforms such as the Raspberry Pi [4] have sparked interest in combining these platforms with open source tools to create inexpensive, easily accessible mobile network penetration testing solutions. This approach would be valuable for education and training purposes as well. However, despite its many advantages the Raspberry Pi faces some limitations on processing power and memory consumption compared with a conventional desktop computer. In this paper, we perform wireless penetration testing using a set of common open source tools on both a desktop and a Raspberry Pi platform, to compare the results and make recommendations for using the Raspberry Pi for education.

For this test, we install the 64 bit version of Kali Linux on both a conventional x86 based desktop (such as the Lenovo X1 Carbon) with 1 MB RAM and on a Raspberry Pi 3 model B. Our testing platforms were equipped with a Panda PAU05 USB wireless adapter [5], which can be configured in transparent mode to capture packets in the $2.4 \mathrm{GHz}$ band from a Netgear router [6]. We set up a WLAN for testing using a standard commercial dual band Cisco wireless router; although our testing was conducted in the $2.4 \mathrm{GHz}$ band, this work should be readily extensible to the $5 \mathrm{GHz}$ band. Our wireless router supports Wi-Fi Protected Setup (WPS) for testing with Reaver, and configuration options for WEP, WPA, and WPA2 encryption [7-10]. While we recognize that WPS contains a known vulnerability and is not recommended for sensitive applications, and that likewise WEP and WPA are not recommended compared with WPA2, we nevertheless find all of these protocols useful when comparing the capabilities of a desktop and mobile platform. Further, all of these protocols are useful for education and training purposes, and unfortunately a surprising number of commercial systems still use these outdated security techniques. For our test network, we manually configured the router with a strong passphrase as recommended by best practice standards for WLANs [1].

The rest of this paper is organized as follows. Section 1 provided the motivation and background for this work. Section II, III, and IV present penetration testing methods and results using Aircrack -ng, Reaver, and Kismet, respectively. Section V describes a comparison between the Desktop and Raspberry Pi environments. Finally, section VI summarizes our results, conclusions, and plans for future research. When this paper references specific command line interface (CLI) instructions, these will be denoted in bold text.

\section{Penetration Testing With AircRACK -NG}

The Aircrack-ng tool is used for the purpose of recovering keys by cracking WEP and WPA/WPA2 keys from captured data packets. Packet capturing is done with the tool aerodump -ng (a sub-tool developed for Aircrack -ng). Aerodump-ng allows any wireless adapter set to monitor mode to scan for wireless networks and lists information that can be used to isolate a network as shown in Figures 1 and 2. Notably, the ESSID is the public identifier of the network, BSSID is the corresponding MAC address of the access point, PWR is the signal strength relative to how close the adapter is to the access point, and lastly the ENC indicates the type of encryption used on the network [8]. We were able to capture packet information and save it in a .cap file from a specified access point via the four-way handshake method. The required command is airodump-ng -bssid (MAC Address) w (filename) (interface). This method requires that a client (labeled as STATION in Figures 3 and 4) is connected to the 
access point which is the authenticator, and from there a deauthentication packet can be sent to the router by issuing the command aireplay-ng $\quad-\mathbf{0} \quad \mathbf{0}-\mathbf{a}$ (bssid) (interface). This command will send a constant de-authentication packet to the access point that causes the router to drop the connection to the client and instead send the PSK/PMK message to the attackers PC, which in turn allows airodump-ng to capture packet information containing the encrypted passphrase as shown in Figure 4.

Aircrack -ng is listed under the category "wireless attacks" within the Kali Linux applications menu, or may be invoked from the CLI directly. As shown in Figure 5, a variety of options are available. The wireless adapter must be set to monitor mode before commencing with any wireless scans with the command airmon-ng start (interface). Once the WPA handshake is successfully captured, the key can be deencrypted by using Aircrack-ng. This tool can access a word list which in turn can be used as a dictionary attack on the .cap file with the encrypted key using the following command; crunch -t -f /usr/share/rainbowcrack/charset.txt | aircrack-ng -w (.cap file with key) -e (SSID name). In this command, Crunch allows a user to access word lists, -t allows you to enter known information about the passcode, $-\mathrm{f}$ calls for the path of the word list. For Aircrack-ng, -w commences the dictionary attack on a specified file, -e calls for the specified SSID name which is required for the key cracking. Furthermore, the attack can range from a few hours to several days depending on the length of the passphrase and the alphanumeric symbols combination. Ultimately, if more information is known about the passphrase prior to the dictionary attack, such as the length, the de-encrypting time will be reduced as shown in Figure 5.

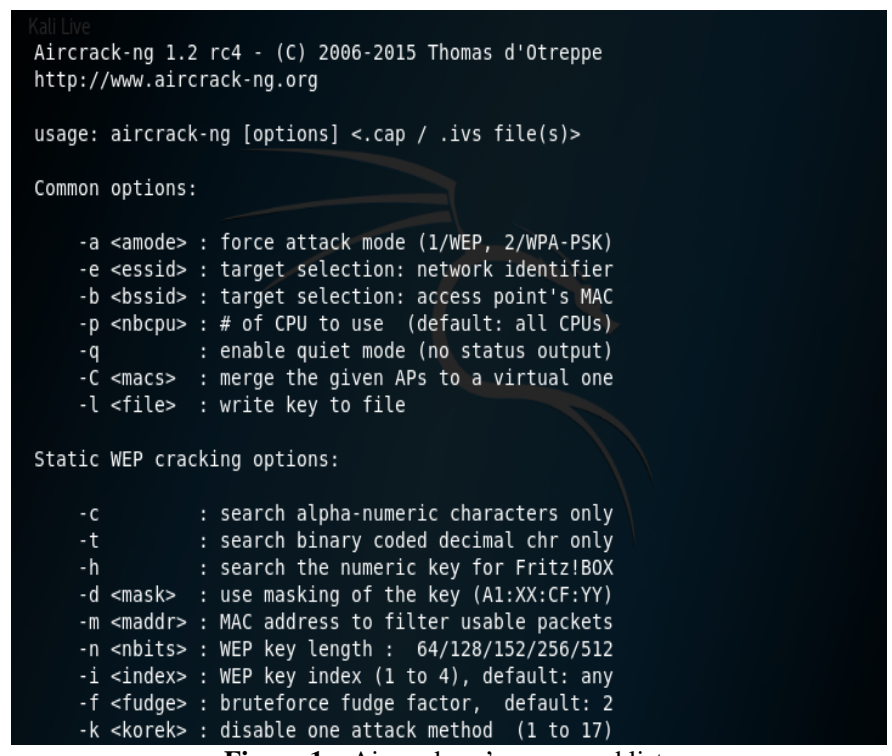

Figure 1 - Aircrack-ng's command list

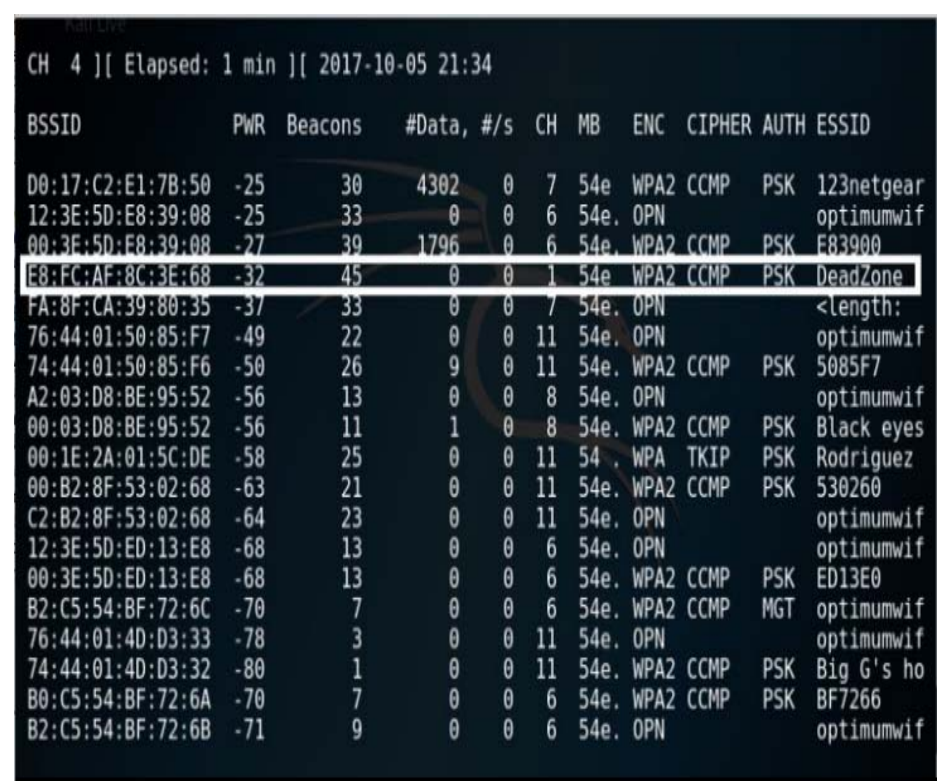

Figure 2 - Results of a scan using Airodump-ng

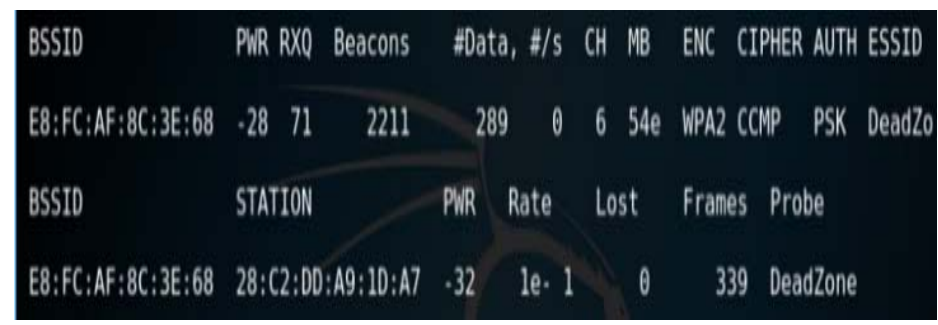

Figure 3 - Isolating the tested network with Airodump-ng

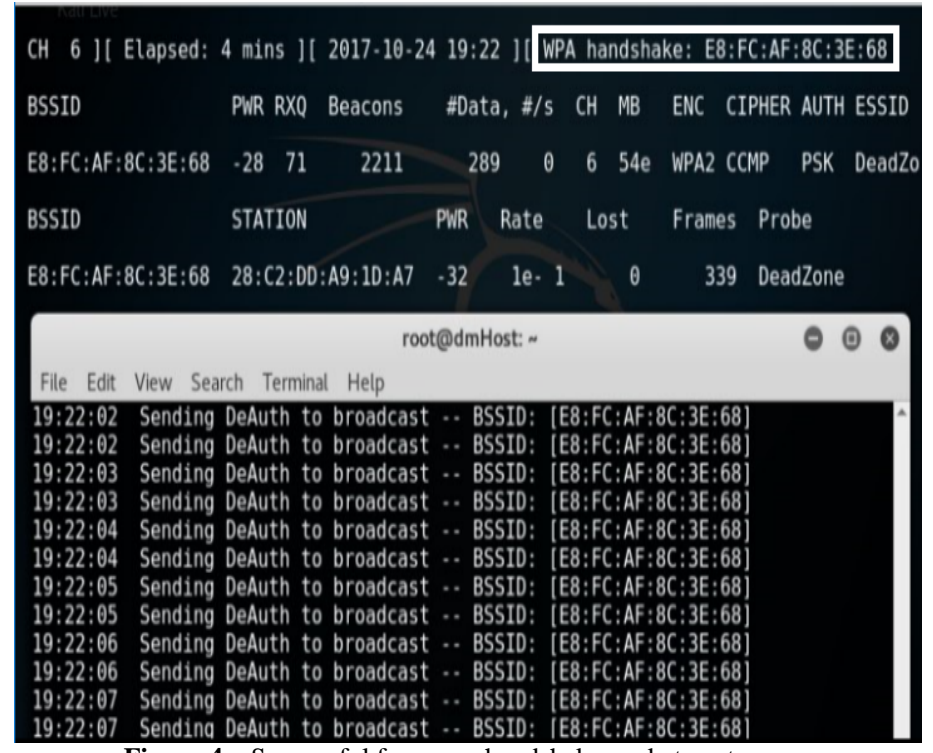

Figure 4 - Successful four-way handshake packet capture

16 ${ }^{\text {th }}$ LACCEI International Multi-Conference for Engineering, Education, and Technology: "Innovation in Education and Inclusion”, 19-21 July 2018, Lima, Peru. 
Aircrack-ng 1.2 rc4

[00:00:38] 46648 keys tested $(1346.35 \mathrm{k} / \mathrm{s})$

KEY FOUND!

Master Key

9A CF 18 BB 5A E5 23 C3 0764 DC CE $09579 C 47$

$522 A 45937 A 13$ B7 039757 C7 4861 DC B2 FB

Transient Key : 7068 C2 7F A7 DB OF 93 B6 B7 F8 47 E2 A9 3F 3D

CO D8 EC 93 CD $4 B \quad 64$ DF OD F8 OD 9E 85 A5 E3 O4

E1 5E 17 2E $3 E 3737$ OE 0317 7B 5A E1 28 8E 9B

C8 D9 OF $7 A$ DC AC 26 gF A9 74 C3 BA 78 6E 3419

EAPOL HMAC O6 B4 15 OD 3C 76 5E 71 E8 DB 3B 3A IB $3 F 95$ 4B

Figure 5 - Completed key de-encryption using Aircrack-ng

\section{Penetration Testing With ReAver}

Reaver is a tool used for performing brute force attacks on a network with WPS enabled. WPS includes an 8-digit passcode that can easily be guessed, and thus provides the passphrase for the WPA/WPA2 decryption. Reaver can be accessed in the applications menu listed under the "Wireless Attacks" category, similarly the tool can also be accessed by using the Reaver command on the CLI as shown in Figure 6. Prior to commencing the commands associated with Reaver, a directory for its content must be created to avoid errors. This is done with the command mkdir /etc/Reaver.

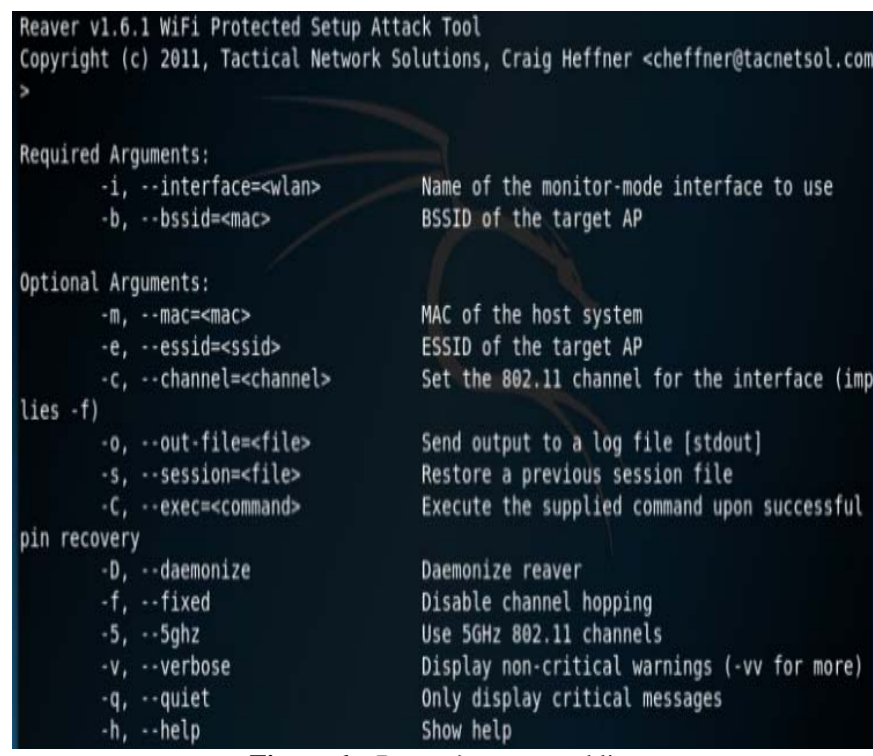

Figure 6 - Reaver's command list

Similar to other tools that issue wireless attacks, the wireless adapter must be set to monitor mode in order to scan for networks with WPS enabled. This is done by using the command airmon-ng start (interface), and after issuing this command tasks such as "Network Manager" must be terminated to reduce subsequent errors. In order to scan for networks with WPS enabled, we use the wash -i wlan0mon command as shown in Figure 7. The results shown provide a list of networks that we are able to perform a brute force attack on with Reaver along with some useful information. ESSID lists the name of the access points, BSSID shows the corresponding MAC address of the devices, $\mathrm{dBm}$ portrays the signal strength in $\mathrm{dB}$, and Lck shows the state of WPS for each access point. A state of "No" means that you are able to perform an attack on the network and vice versa.

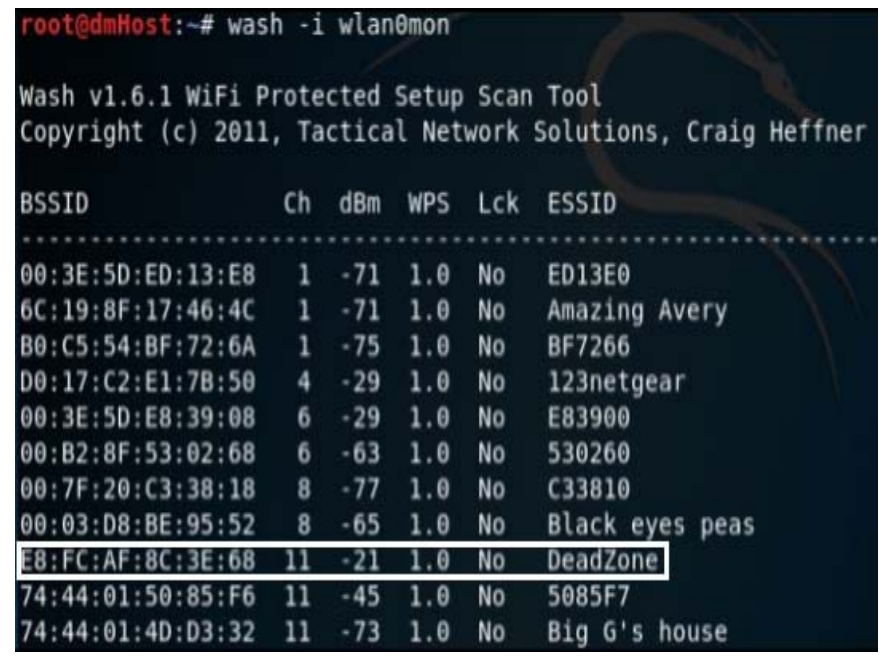

Figure 7 - List of wireless networks with WPS enabled discovered by Reaver

Notably, a brute force attack with Reaver may encounter several errors during the process, such as a "Failed to associate" error and similarly, a "Detected AP rate limiting" warning. The former is related to signal strength, more specifically the weaker the signal strength the harder it is to guess the WPS pin and unveil the passcode. The latter occurs when the router locks itself due to reaching the max limit of guesses for the pin. The lock is removed after a certain amount of time has passed and Reaver can resume the attack. Lastly, as mentioned Reaver has the option to resume a saved session which means that the pins that have already been used as guesses will not be reused. Relative to uncovering the WPA/WPA2 passcode, the longer Reaver is able to attack the same access point the more likely the passcode will be obtained and access to the network will be granted.

\section{Penetration Testing With Kismet}

Kismet is a tool that is able to perform network scans with the option to collect packet information and attempt data decryption. Kismet can be accessed within the Kali Linux applications menu within the "Wireless Attacks" category; issuing the command kismet on the CLI results in the initial default screen shown in Figure 8. Before initiating a scan, Kismet provides configuration options including whether to

$16^{\text {th }}$ LACCEI International Multi-Conference for Engineering, Education, and Technology: "Innovation in Education and Inclusion”, 19-21 July 2018, Lima, Peru. 
run Kismet as root, whether to configure a remote Kismet server, and whether to enable logging or console modes.

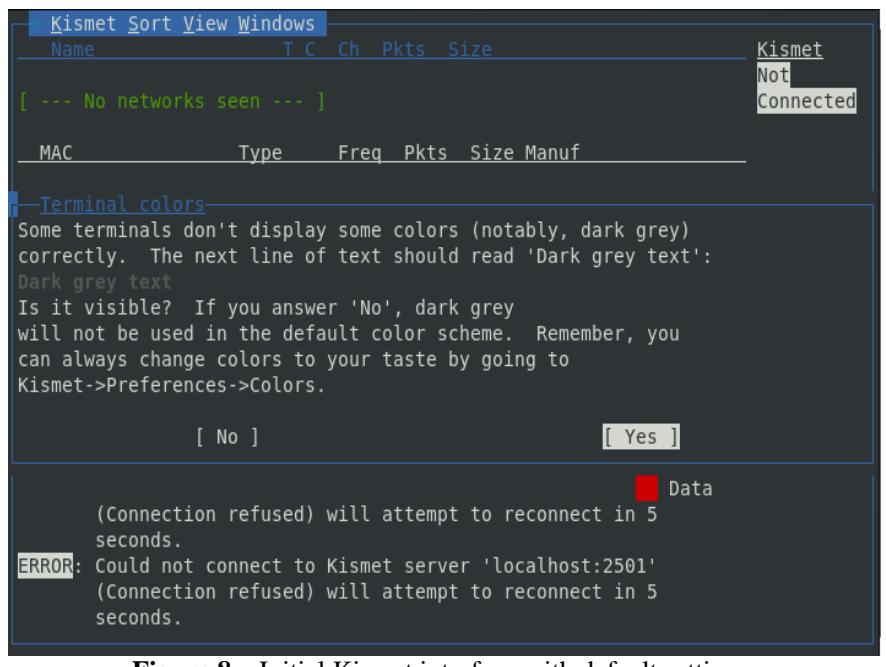

Figure 8 - Initial Kismet interface with default settings

Once a target interface is specified (i.e. WLAN adapter name), Kismet can run a packet capture scan on nearby WLAN networks. It is possible to apply a "channel lock" option, which means that Kismet will only listen to traffic from the access point associated with a specific channel. This can dramatically reduce the time required to analyze captured packets. There are other packet filtering options available, including sorting by SSID, BSSID (MAC address), and more. As shown in Figure 9, a basic Kismet scan reveals the type of encryption used by the target access point, device manufacturer, and other useful details [10].

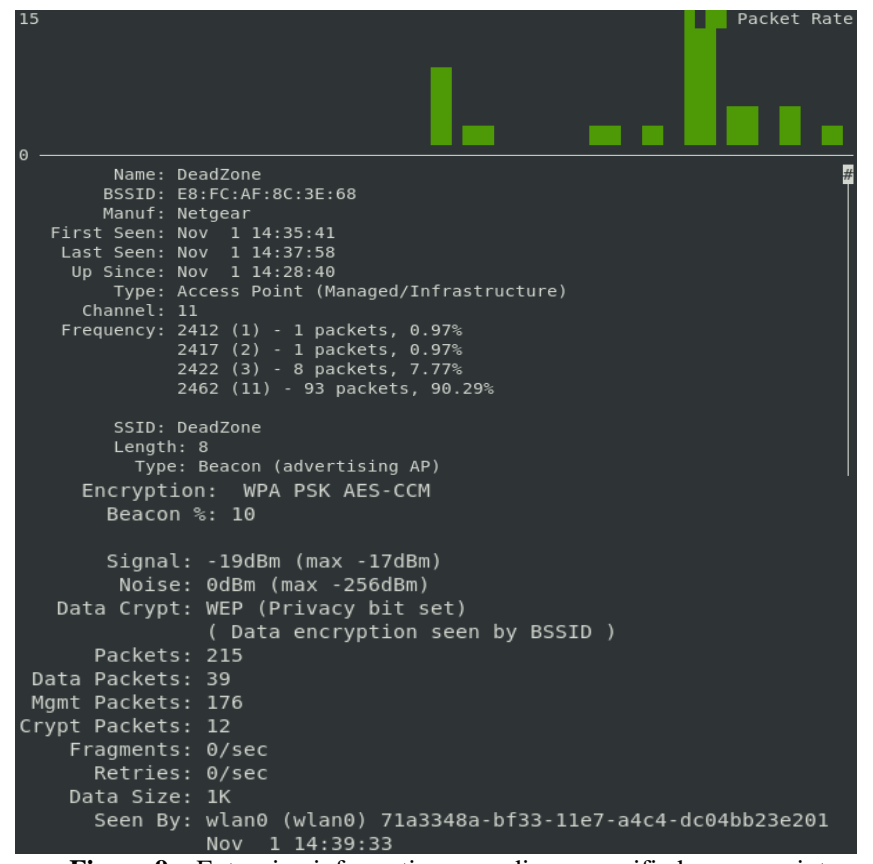

Figure 9 - Extensive information regarding a specified access point
Kismet can perform passive packet sniffing, and detect basic attacks such as ARP floods or malformed packets. By capturing the four-way handshake during device association and the encrypted EAPoL key, Kismet allows us to collect the data required to decrypt wireless authentication keys in a manner similar to WireShark. This is shown in Figure 10. This can be done using tools such as Aircrack -ng to perform a brute force dictionary attack on the packet capture file (filetype .cap) created by Kismet.

\begin{tabular}{|c|c|c|c|c|c|}
\hline \multicolumn{4}{|c|}{ Kismet-20171101-15-19-23-1.pcapdump } & $\boldsymbol{\theta}$ & 8 \\
\hline \multicolumn{6}{|c|}{ File Edit View Go Capture Analyze S- Statistics Telephony Wireless Iools $\underline{\text { Help }}$} \\
\hline \multicolumn{6}{|c|}{ 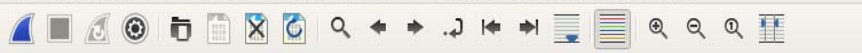 } \\
\hline 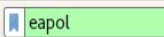 & & & $x \Rightarrow$ & Expression... & + \\
\hline Time & Source & Destination & Protocol & Length Info & \\
\hline 150452.941339 & Netgear_4d:d3:32 & Apple_59:c8:36 & EAPOL & 187 Key & (Mes \\
\hline 151252.959889 & Netgear_4d:d $3: 32$ & Apple_59:c8:36 & EAPOL & 245 Key & (Mes \\
\hline 321873.872517 & Netgear $8 \mathrm{c}: 3 \mathrm{e}: 68$ & Microsof $44: \mathrm{d} 1: \mathrm{c} 8$ & EAPOL & 187 Key & (Mes \\
\hline 322373.902130 & Netgear $8 c: 3 e: 68$ & Microsof $44: \mathrm{d} 1: \mathrm{c} 8$ & EAPOL & $221 \mathrm{Key}$ & (Mes \\
\hline 322973.906489 & Microsof_44:d1:c8 & Netgear_8c:3e:68 & EAPOL & 165 Key & (Mes \\
\hline 10556117.836607 & Netgear_8c:3e:68 & Apple_2a:2c:dc & EAPOL & $187 \mathrm{Key}$ & (Mes \\
\hline 10560117.839520 & Apple_2a:2c:dc & Netgear_8c:3e: 68 & EAPOL & $187 \mathrm{Key}$ & (Mes \\
\hline 10565117.855596 & Apple_2a:2c:dc & Netgear_8c:3e:68 & EAPOL & 165 Key & (Mes \\
\hline
\end{tabular}

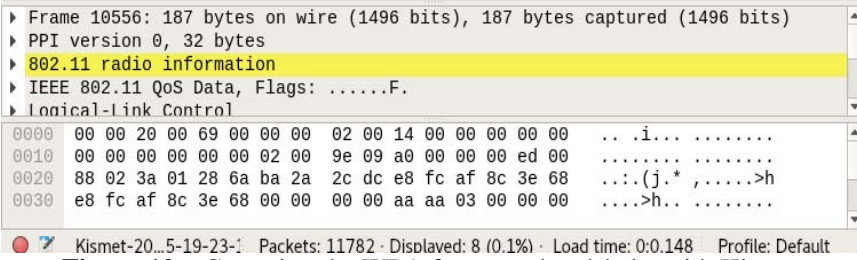
Figure 10 - Capturing the WPA four-way handshake with Kismet

\section{RASPBERRY PI TEST COMPARISON}

We compared the use of Aircrack -ng, Reaver, and Kismet running on both an x86 desktop client and on the Raspberry Pi 3. Both environments were able to load and run the Kali Linux toolkit, allowing similar testing to be performed on a target WLAN. For example, using Aircrack -ng both platforms were effective, and in some cases the Raspberry Pi 3 was actually slightly faster than the desktop, completing capture of the WPA four way handshake in only a few seconds as shown in Figure 11.

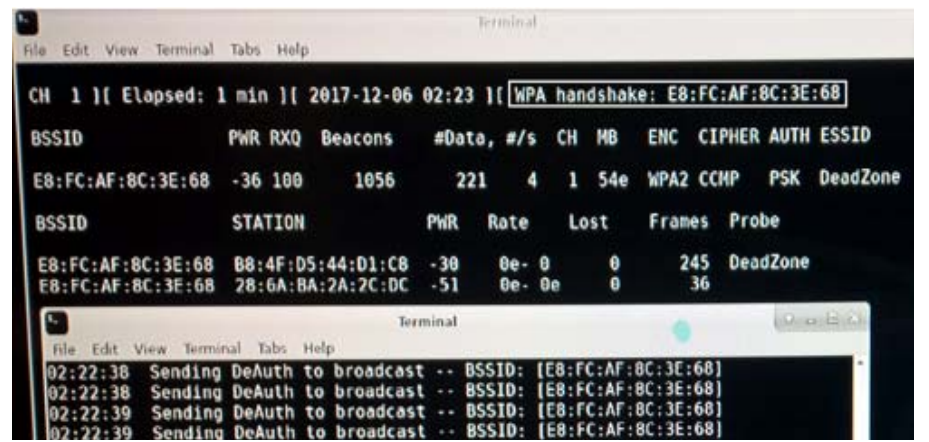

Figure 11 - Successful four-way handshake packet capture with Airodumpng (Raspberry Pi)

16 ${ }^{\text {th }}$ LACCEI International Multi-Conference for Engineering, Education, and Technology: "Innovation in Education and Inclusion”, 19-21 July 2018, Lima, Peru. 
A more significant performance difference was observed when conducting processor or memory intensive penetration tests such as dictionary attacks. These tests were significantly faster using the desktop environment (seconds vs minutes on the mobile platform). We note that the mobile platform could still launch dictionary attacks fast enough to be viable, just not as rapidly as the desktop platform. For testing in situ the mobile platform has clear advantages, although total processing time was minimized by using the mobile platform to capture packets and the desktop environment to perform offline password cracking.

Of the three tools we evaluated, only Reaver was unable to perform reasonably on the Raspberry Pi 3. While it was still possible to scan for wireless networks using Reaver, attempting brute force cracking resulted in repeated timeouts and failed attempts, as shown in Figure 12.

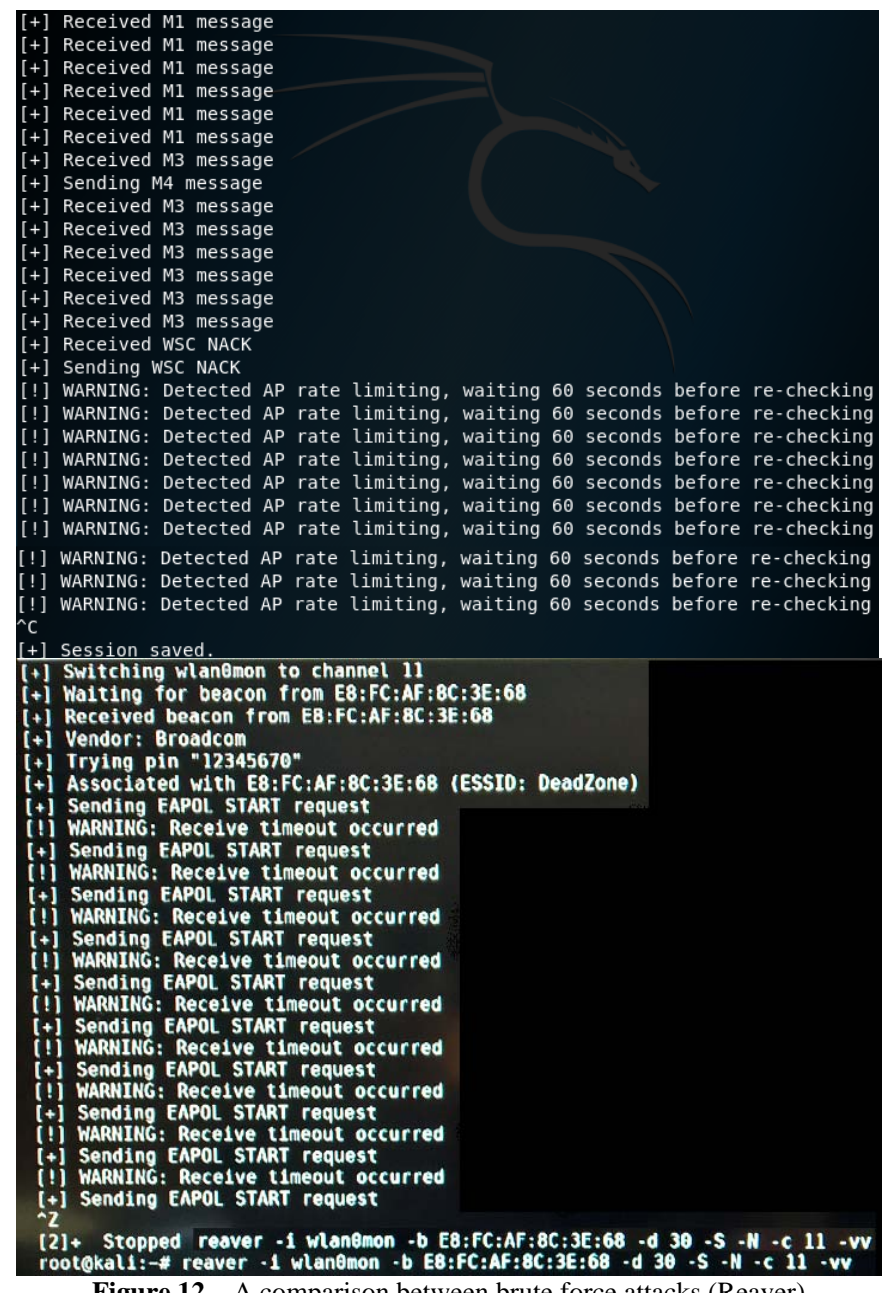

Figure 12 - A comparison between brute force attacks (Reaver)

Lastly, we found no performance difference when running Kismet on a desktop vs a mobile platform. Both variations allowed for proper packet sniffing and capture of the encrypted EAPoL keys. Also, we were able to run WireShark in both desktop and mobile platforms with no discernable performance impact.

\section{CONCLUSIONS}

This paper has demonstrated introductory WLAN penetration techniques using Aircrack -ng, Reaver, and Kismet which are suitable for an introductory cybersecurity education program. We then compared these approaches using a conventional desktop x86 compute platform and a mobile Raspberry Pi 3 platform. Use cases such as passive wireless network packet sniffing (particularly during the fourway association handshake), and password decryption using WEP, WPA, and WPA2 were evaluated. Both online and offline password cracking was compared using brute force dictionary attacks. We also studied the known WPS identification code vulnerability. Our results show that while Aircrack-ng and Kismet were both viable penetration testing tools on the Raspberry $\mathrm{Pi}$, Reaver encountered significant issues which rendered it unusable. Specifically, Reaver was unable to complete online password cracking on the Raspberry Pi due to limitations in CPU performance and memory usage. Future research may include studying the use of compute clusters to improve performance during WLAN penetration testing, which may allow us to overcome the limitations of using Reaver on the Raspberry Pi.

\section{REFERENCES}

[1] J. Broad and A. Binder, Hacking with Kali: Practical Penetration Testing Techniques, Waltham, Massachusetts : Syngress, 2014.

[2] Z. Trabelsi, K. Hayawi, A. Braiki, and S. Mathew, Network Attacks and Defenses: A Hands-on Approach, Boca Raton, Florida: CRC Press, 2013.

[3] "Category: Wireless Attacks," Penetration Testing Tools. [Online]. Available: https://tools.kali.org/wireless-attacks/.[Accessed:Sept. 2017].

[4] V. Ramachandran and C. Buchanan, Kali Linux Wireless Penetration Testing Beginner's Guide: Master Wireless Testing Techniques to Survey and Attack Wireless Networks With Kali Linux, Birmingham, England: Packt Publishing, 2015.

[5] “Top Wardriving USB adapters,” WirelessSHack. [Online]. Available http://www.wirelesshack.org/top-wardriving-usb-adapters.html. [Accessed: Sep. 2017].

[6] "NETGEAR N900 Wireless Dual Band Gigabit Router: Model WNDR4500v2 User Manual, " [Online]. Available: www.downloads.netgear.com/files/GDC/WNDR4500V2/WN DR4500v2 UM 25Aug2014.pdf. [Accessed: Sept. 2017].

[7] A. Carranza, J. Magallanes, C. DeCusatis, and J. Espinal, “Automated Wireless Network Penetration Testing Using Wifite and Reaver,” Boca Raton, Florida: LACCEI, 2017

[8] “Aircrack-ng," airodump-ng [Aircrack-ng]. [Online]. Available: $\quad$ https://www.aircrack-ng.org/doku.php?id=airodumpng.[Accessed: Oct2017].

[9] W. by S. DeLeeuw, “Home,” How To Crack WPA/WPA2 (2012) SmallNetBuilder.[Online].https://www.smallnetbuilder.com/wireless/wire less-howto/31914-how-to-crack-wpa-wpa2-2012. [Accessed: Oct. 2017]

[10]"Using Kismet To Analyze Wi-Fi Access Points and Their Client Computers,” Tucson Computer Society [Online]. Available: http://aztcs.org/meeting_notes/winhardsig/ kismet/kismet.htm. [Accessed: 01-Nov-2017].

16 ${ }^{\text {th }}$ LACCEI International Multi-Conference for Engineering, Education, and Technology: "Innovation in Education and Inclusion”, 19-21 July 2018, Lima, Peru. 